\title{
Shoulder Joint Tendons
}

National Cancer Institute

\section{Source}

National Cancer Institute. Shoulder Joint Tendons. NCI Thesaurus. Code C161387.

The tendons that connect the muscles and bones that comprise the glenohumeral and acromioclavicular joints and enable abduction of the arm and stabilization of the shoulder. 